\title{
Planejamento nutricional da alta hospitalar: breve revisão da literatura e proposta de instrumento de avaliação
}

\author{
Nutritional planning of hospital discharge: a brief literature review and proposal for an assessment \\ instrument
}

DOI: $10.37111 /$ braspenj.AE202035402

Rodrigo Costa Gonçalves

Maria Carolina Gonçalves Dias ${ }^{2}$

Nara Lucia Andrade Lopes Segadilha ${ }^{3}$

Ana Cristina Schmidt de Oliveira-Netto ${ }^{4}$

Maria do Socorro Lira Paes Batista ${ }^{5}$

Maria de Lourdes Teixeira da Silva ${ }^{6}$

\begin{abstract}
Unitermos:
Desnutrição. Avaliação nutricional. Tempo de internação. Nutrição. Alta do Paciente. Readmissão do Paciente. Equipe de Assistência ao Paciente. Cuidado Transicional.
\end{abstract}

\section{Keywords:}

Malnutrition. Nutrition assessment. Length of stay. Nutrition. Patient Discharge. Patient readmission. Patient Care Team. Transitional Care.

\section{Endereço para correspondência}

Rodrigo Costa Gonçalves

Avenida 136, 761, sala A- 46 - Setor Sul - Goiânia, GO, Brasil - CEP: 74093-250

Email: nutrir.terapianutricional@gmail.com

\section{Submissão}

17 de julho de 2020

\section{Aceito para publicação}

15 de dezembro de 2020

\begin{abstract}
RESUMO
Introdução: Em virtude de sua frequência e consequências, a desnutrição é uma condição preocupante no âmbito hospitalar, especialmente no caso de adultos internados em unidades de terapia intensiva e outros de alto risco, estando associada a maior risco de morbidade e mortalidade, aumento do tempo de internação, maior frequência de reinternações e aumento de custos. Embora a falta de planejamento para a alta hospitalar possa agravar essas consequências, ainda não há diretrizes para planejamento nutricional da alta hospitalar que possam ser adotadas em ampla escala no Brasil. Método: Um painel de especialistas foi reunido com o intuito de avaliar os tópicos mais relevantes na literatura relacionada ao planejamento nutricional da alta hospitalar, discutir sua experiência a esse respeito e propor um instrumento que possa nortear e justificar a importância da alta nutricional planejada e segura. Resultados: $O$ plano de alta organizado traz benefícios clínicos e nutricionais para o paciente, além de vantagens para os familiares e o serviço de saúde. $O$ cuidado nutricional e uma melhor comunicação e orientação da equipe multiprofissional preparam o paciente e seus familiares para reduzir o tempo de internação e evitar reinternação. O planejamento de alta requer o desenvolvimento de um plano individualizado, bem como a educaçáo do paciente, familiares e cuidadores, sendo o plano de cuidados nutricionais inserido no plano de alta. Com base nestas considerações, propomos um instrumento que tem por objetivo sistematizar a alta nutricional por meio da coleta das informações mais relevantes ligadas ao risco nutricional e à abordagem terapêutica desse risco, normatizando a comunicação com o paciente, seus cuidadores e a equipe de saúde. Conclusões: $O$ instrumento aqui apresentado deverá ser testado na prática clínica, e espera-se que ele possa permitir melhor acompanhamento da jornada do paciente, conduzindo-o para a alta hospitalar bem-sucedida.
\end{abstract}

\section{ABSTRACT}

Introduction: Due to its frequency and potential consequences, malnutrition is a worrisome condition in the hospital environment, especially in the case of adults admitted to intensive care units and others at high risk; malnutrition is associated with a higher risk of morbidity and mortality, prolonged hospital stay, higher frequency of readmissions, and increased costs. Although the absence of a planning for hospital discharge can aggravate these consequences, there are no guidelines for nutritional planning for hospital discharge that can be widely adopted in Brazil. Methods: A panel of experts was convened to assess the most relevant topics in the literature related to hospital discharge planning, discuss their experience in this regard, and propose an instrument that could guide and justify the importance of planned and safe nutrition discharge. Results: An organized and explicit discharge plan brings clinical and nutritional benefits to the patient, as well as advantages for family members and the health-care service. Nutritional care and better communication and guidance from the multiprofessional team prepare the patient and family members to reduce length of hospital stay and may avoid readmissions. Discharge planning requires the development of an individualized plan, as well as the education of the patient, family and caregivers; the nutritional care plan is inserted in the discharge plan. Based on these considerations, we propose an instrument that aims to systematize the nutritional discharge through the collection of the most relevant information related to the nutritional risk and the therapeutic approach to this risk, standardizing the communication with the patient, their caregivers, and the health-care team. Conclusions: The instrument presented here should be tested in clinical practice, and it is hoped that it can allow a better follow-up of the patient's journey, leading to a more successful hospital discharge.

1. Médico; Especialista em Medicina Intensiva, Nutrologia e Nutrição Parenteral e Enteral; Hospital de Urgências da Região Noroeste de Goiânia - HUGOL, Hospital Órion, Goiânia, GO, Brasil.

2. Nutricionista chefe da Divisão de Nutrição e Dietética do Instituto Central (IC-HC) do Hospital das Clínicas da Faculdade de Medicina da Universidade de São Paulo (HCFMUSP), Coordenadora Administrativa da EMTN-ICHC, Mestre em Nutrição Humana pela Universidade de São Paulo, Especialista em Nutrição Parenteral e Enteral pela BRASPEN, São Paulo, SP, Brasil.

3. Coordenadora Administrativa e Nutricionista Clínica da EMTN do Hospital Copa D’Or; Mestre em Nutrição Clínica pela Universidade Federal do Rio de Janeiro; Especialista em Nutrição Parenteral e Enteral pela BRASPEN, Rio de Janeiro, RJ, Brasil.

4. Médica Intensivista; Mestre em Medicina Interna pela Universidade Federal do Paraná (UFPR); Especialista em Nutrição Parenteral e Enteral pela BRASPEN; Complexo Hospital de Clínicas UFPR / Hospital das Nações, Curitiba, PR, Brasil.

5. Coordenadora técnica e nutricionista da EMTN da Santa Casa de Misericórdia de Maceió; Especialista em nutrição parenteral e enteral pela BRASPEN; Especialista em nutrição clínica e terapêutica nutricional pela UNIGUAÇU; Santa Casa de Misericórdia de Maceió, Maceió, AL, Brasil.

6. Mestre em Gastroenterologia; Especialista em Nutrição Parenteral e Enteral; Diretora do Ganep - Nutrição Humana; Coordenadora das EMTNs do Hospital Beneficência Portuguesa de São Paulo e Mirante, São Paulo, SP, Brasil. 


\section{INTRODUÇÃO}

Em virtude de sua frequência e possíveis consequências, a desnutrição é uma condição particularmente preocupante no ambiente hospitalar, afetando entre $20 \%$ e $60 \%$ dos pacientes adultos internados por diferentes condições clínicas ${ }^{1-3}$. Nesse âmbito, a desnutrição está associada a maior risco de morbidade e mortalidade, aumento do tempo de internação, maior frequência de reinternações e aumento de custos $^{3-6}$. A preocupação é ainda mais evidente no caso de adultos internados em unidades de terapia intensiva (UTIs), onde a desnutrição acomete entre $40 \%$ e $80 \%$ dos pacientes e é tanto causa quanto consequência de uma série de alterações fisiopatológicas associadas a piores desfechos ${ }^{3,7-9}$. Em um estudo brasileiro recente, a piora do estado nutricional na primeira semana de hospitalização foi identificada como fator preditivo de maior tempo de hospitalização e de ocorrência de reinternação no período de 6 meses após a alta, ressaltando a importância do monitoramento nutricional no cuidado $^{10}$. Nesse estudo, o declínio de categoria na Avaliação Subjetiva Global (ASG, Subjective Global Assessment) foi associado a aumento de 2 vezes na chance de internação prolongada, e de 3,6 vezes de readmissão aos 6 meses $^{10}$.

Assim, a atenção a aspectos nutricionais em resposta a doenças agudas e crônicas deve receber ênfase progressiva durante a trajetória do paciente em sua totalidade, incluindo a alta hospitalar programada ${ }^{11-14}$. A relevância de tal atenção fica ainda mais evidente quando se considera que cerca de $20 \%$ a $45 \%$ dos pacientes apresentam desnutrição no momento da alta ${ }^{15}$. Sabe-se que, na prática clínica, o processo de alta hospitalar é comumente feito de maneira pouco planejada e padronizada, e que tal falta de planejamento pode contribuir para a alta precoce, em que o paciente é liberado antes de alcançar as condições ideais para a alta, o aumento do risco de piora nutricional, o prolongamento da internação e aumento de complicações pós-alta e do número de reinternações não planejadas, além de acarretar maiores custos ao serviço de saúde e pior qualidade de vida ao paciente ${ }^{11,16}$.

Diversos estudos demonstraram os benefícios de se estabelecer precocemente planos de alta e de cuidado estruturados e individualizados, baseados nas necessidades e condições clínicas e nutricionais dos pacientes. Em uma meta-análise de 30 estudos randomizados, pacientes alocados para uma estratégia de cuidado com planejamento de alta individualizado tiveram redução do tempo de hospitalização e da taxa de reinternação quando comparados a pacientes que receberam cuidado padrão, sem plano de alta individual ${ }^{17}$. Naylor et al. ${ }^{18}$ relataram que pacientes hospitalizados, com idade igual ou superior a 65 anos, alocados para uma intervenção envolvendo um planejamento de alta mais sistematizado e com seguimento domiciliar pós-alta, tiveram menor taxa de hospitalização $(37,1 \%$ vs. $20,3 \%)$, de múltiplas reinternações (6,2\% vs. $20,3 \%)$, menor duração da internação (1,53 vs. 4,09 dias) e aumento do tempo até a reinternação, do que pacientes do grupo controle, submetidos ao procedimento de alta padrão. Em um estudo randomizado com pacientes portadores de doença pulmonar crônica obstrutiva, Lainscak et al. ${ }^{19}$ também mostraram os benefícios de uma intervenção de alta coordenada, visto que pacientes que receberam a intervenção tiveram menor taxa de hospitalização pela doença ( $14 \%$ vs. $31 \%$ ) e por qualquer causa ( $31 \%$ vs. $44 \%$ ) do que pacientes em cuidado padrão.

As etapas necessárias para um planejamento adequado da alta vem sendo cada vez mais discutidas na literatura ${ }^{11,13,16,20}$. As recomendações preconizam que o planejamento da alta seja iniciado de maneira precoce, para que sejam identificadas as barreiras e tomadas as devidas ações, utilizando uma abordagem multidisciplinar, com um coordenador, e acompanhamento pós-alta. Um posicionamento da ANMCO listou dez etapas para um planejamento adequado de $a \mathrm{ta}^{16}$. Um dos pontos a serem considerados é que, nesse processo de planejamento, a alta não é vista como um evento isolado, mas como um processo de continuidade do cuidado e que depende do engajamento do paciente/cuidador, e exige comunicação e ação coordenada da equipe responsável pelo cuidado para garantir o tratamento nutricional e acompanhamento adequados ${ }^{16}$.

Apesar da existência de diretrizes internacionais e de orientações locais em determinadas instituições de nosso País, ainda não há consenso sobre um instrumento de planejamento de alta que possa ser aplicado em ampla escala no Brasil, especialmente no que diz respeito ao planejamento nutricional da alta hospitalar de adultos. Assim, um painel multidisciplinar de especialistas médicos e nutricionistas foi reunido com o intuito de avaliar os tópicos mais relevantes na literatura relacionada ao planejamento nutricional da alta hospitalar, discutir sua experiência a esse respeito e propor um instrumento que possa ser usado pela equipe de nutrição responsável pelo cuidado hospitalar, para nortear e justificar a importância da alta nutricional planejada e segura. $\bigcirc$ presente artigo relata o resultado desse trabalho.

\section{MÉTODO}

\section{Constituição e Objetivos do Painel}

O painel, constituído pelos presentes autores, reuniu-se em três ocasiões distintas nos anos de 2018 e 2019, com apoio financeiro da Danone Nutrícia. $O$ presente artigo é de responsabilidade exclusiva do painel, que contou com apoio editorial da empresa Dendrix (São Paulo), financiado pela Danone Nutricia.

Os objetivos do painel foram discutir a jornada do paciente adulto em uso de terapia nutricional, desde a UTI até a alta hospitalar, abordando as dificuldades da transição da UTI para enfermaria e estratégias para reabilitação nutricional a médio e longo prazos. Além disso, o painel teve por objetivo elaborar um instrumento para auxílio no planejamento de alta em terapia nutricional segura, especialmente para uso em instituições brasileiras. 


\section{Revisão da Literatura}

Foi feita uma revisão narrativa da literatura pertinente, com base nos temas discutidos e em apresentações feitas por membros do painel, tentando identificar, sempre que possível, artigos com maior relevância para o cenário brasileiro. $\bigcirc$ objetivo da revisão foi o de reunir a evidência científica que pudesse justificar e servir de base para criação do instrumento para planejamento da alta nutricional.

\section{TÓPICOS RELEVANTES DISCUTIDOS PELO PAINEL PARA CRIAÇÃO DO INSTRUMENTO}

\section{Prevalência de Desnutrição em Pacientes Brasilei- ros Hospitalizados e Importância do Rastreamento Precoce}

No Brasil, o estudo epidemiológico IBRANUTRI encontrou altas taxas de prevalência de desnutrição e desnutrição grave de $48,1 \%$ e $12,6 \%$, respectivamente, em pacientes adultos hospitalizados no sistema público por causas variadas ${ }^{21}$. Os autores relataram, também, que menos de $20 \%$ dos pacientes tinham algum registro de informações sobre o estado nutricional em seus prontuários, e apenas 7,3\% dos casos fizeram uso de terapia nutricional. Esses achados de alta prevalência de desnutrição, pouca conscientização sobre o problema da desnutrição entre os médicos e a subprescrição de terapias nutricionais também foram encontrados em estudos mais recentes, incluindo uma revisão sistemática envolvendo dados de cerca de 30.000 pacientes hospitalizados em 12 países latino-americanos em que a taxa de desnutrição na admissão variou entre $40 \%$ e $60 \% 3$. Pinho et al. ${ }^{22}$ observaram uma prevalência de desnutrição entre pacientes brasileiros com câncer internados em hospitais públicos de 36,1\% a $55 \%$, dependendo da faixa etária, e recomendaram que o screening e a avaliação nutricional sejam realizados imediatamente após a hospitalização, para possibilitar o diagnóstico e intervenções precoces. Apesar da alta frequência de desnutrição entre pacientes hospitalizados, essa condição permanece subidentificada e subtratada. Um estudo retrospectivo com mais de 8.000 pacientes desnutridos, encontrou que apenas 3,1\% destes receberam suplementação oral. Quando comparados aos pacientes que não receberam, aqueles que usaram suplementação nutricional oral tiveram $38,8 \%$ menos reinternações, sendo essa redução ainda maior $(46,1 \%)$ em doentes oncológicos. Além disso, observou-se uma redução na duração da hospitalização conforme reduzia o intervalo entre a admissão e o início da suplementação oral ${ }^{23}$.

Embora sabidamente associado a desfechos desfavoráveis durante a internação hospitalar, o diagnóstico da desnutrição, muitas vezes, não é feito por ocasião da admissão e até durante a internação hospitalar ${ }^{24}$. É possível que tal falha seja devida a conhecimento insuficiente por parte de profissionais de saúde, uma vez que diversos estudos documentaram esse fenômeno entre médicos, enfermeiros e até mesmo nutricionistas ${ }^{25-28}$. Por outro lado, é possível que outros fatores contribuam para a falta de reconhecimento de casos de desnutrição; tais fatores incluem a incerteza sobre atribuição de responsabilidades entre diferentes profissionais e setores do hospital, a falta de coordenação das atividades entre diferentes serviços e a ausência de instrumentos que possam ser usados para avaliar objetivamente o problema e guiar suas possíveis soluções em cada instituição ${ }^{29,30}$.

A importância da atenção a aspectos nutricionais em adultos internados é redobrada quando se considera que a prevalência de desnutrição tende a aumentar conforme aumenta o período de internação, tornando crucial o seu reconhecimento e a intervenção terapêutica precoce ${ }^{3,21}$. $\bigcirc$ problema é ainda maior quando se consideram pacientes idosos, muitos dos quais em mais alto risco nutricional do que indivíduos mais jovens em função da frequente combinação de ingestão alimentar reduzida e da presença de comorbidades $^{31,32}$. Da mesma forma, pacientes internados em UTIs e aqueles com características especiais, tais como câncer, doença gastrintestinal de tratamento cirúrgico, politrauma e outros, comumente apresentam risco nutricional elevado ${ }^{33}$.

Evidentemente, o rastreamento e a avaliação inicial do paciente desnutrido devem ser seguidos por estratégias de intervenção e acompanhamento, conforme delineado pela iniciativa feedM.E. (Medical Education) Global Study Group ${ }^{34}$ e discutido em maiores detalhes adiante.

Diante do cenário atual, a campanha 'Diga não à desnutrição' foi criada pela Sociedade Brasileira de Nutrição Parenteral e Enteral (BRASPEN) com o objetivo de alertar sobre o problema da desnutrição hospitalar e suas consequências, bem como discutir estratégias e ações práticas voltadas para o aprimoramento do rastreamento, diagnóstico, avaliação e manejo da desnutrição, que possam impactar positivamente e reduzir a prevalência de desnutrição e suas consequências ${ }^{35}$. Dentre os 11 passos estabelecidos pela campanha para o combate à desnutrição, está a orientação à alta hospitalar ${ }^{35}$.

Planejamento Nutricional da Alta Hospitalar: Importância, Estratégias e Barreiras Previsíveis

Como mencionado, a alta hospitalar é frequentemente mal planejada e feita de modo não padronizado, o que pode causar descontinuidade e fragmentação do cuidado dispensado ao paciente e colocá-lo sob maior risco após a alta. Assim, o planejamento da alta pode ser entendido como a tentativa de reduzir o tempo de internação hospitalar e reinternação precoce ou não planejada e melhorar a coordenação dos serviços após a alta ${ }^{17,35}$. O plano de alta organizado traz benefícios clínicos e nutricionais para o paciente, além de vantagens para os familiares e o serviço de saúde ${ }^{16,36}$. O maior envolvimento e dedicação da equipe multiprofissional atua como facilitador da transição entre o período de hospitalização e o domicílio, e, dessa forma, aumenta a segurança da alta hospitalar. $O$ cuidado nutricional efetivo, a melhor comunicação e orientação da equipe 
multiprofissional preparam o paciente e seus familiares para reduzir o tempo de internação e evitar reinternação.

Portanto, contrastando com a alta não planejada, em que o tempo de hospitalização é todo dedicado ao tratamento, sem ações voltadas para a alta e com a educação do paciente acontecendo só ao final da hospitalização, quando se instala um procedimento de planejamento de alta, as ações de engajamento e educação do paciente/cuidador são feitas desde o início, com o intuito de se garantir que a transição seja realizada da maneira mais gradual, segura e consciente, bem como de acordo com as necessidades individuais. Nesse caso, a equipe já trabalha desde o início com uma data estimada para a alta, que vai sendo avaliada e ajustada durante a internação ${ }^{16}$. Alguns instrumentos e algoritmos com sugestões sobre como desenvolver um protocolo de alta para a transição de cuidados UTI - unidade de internação estão disponíveis na literatura. Em um estudo de mapeamento de intervenção, a importância de uma comunicação efetiva e de materiais de suporte na alta foi ressaltada nas entrevistas com pessoas que passaram por internação em UTI e familiares considerados com grande experiência em UTI. A necessidade de maior informação e conhecimento sobre sintomas de Síndrome Pós Cuidado Intensivo (Post Intensive Care Sindrome - PICS) e delirium foi também apontada e reconhecida ${ }^{20}$. O estudo RECOVER ${ }^{36}$ comparou as experiências de pacientes submetidos a uma intervenção complexa de cuidados pós-UTI com intensificação de fisioterapia, cuidado nutricional e fornecimento de informações versus a experiência de pacientes acompanhados com o cuidado padrão, demonstrando que a intervenção melhorou significativamente a satisfação dos pacientes com a reabilitação.

O registro das informações clínicas relevantes, da evolução e de procedimentos realizados e em andamento é fundamental para o acompanhamento do paciente e para garantir a continuidade do cuidado, tanto na transição entre unidades do hospital como na transição para casa, dessa forma reduzindo os riscos associados à fragmentação ou interrupção dos cuidados ${ }^{35,37}$. Sabe-se que os momentos de transição de cuidado, seja entre instituições, unidades de atendimento da mesma instituição ou para casa, são uma fonte importante de problemas, como erros médicos, eventos adversos, ineficiência, custos desnecessários e insatisfação dos pacientes, e tais problemas podem ser mitigados por um melhor registro e comunicação entre as partes. Em pacientes críticos transferidos para outros hospitais, foi observada uma associação entre a quantidade de informações disponíveis no documento de transferência e a melhora de desfechos clínicos. Pacientes com documentações mais completas apresentaram redução significativa na mortalidade hospitalar, ocorrência de eventos adversos e duplicação de trabalho ${ }^{37}$.

Do ponto de vista nutricional, o planejamento de alta consiste no desenvolvimento de um plano individualizado, bem como na educação e na maior conscientização da necessidade do cuidado do paciente, familiares e cuidadores ${ }^{35}$.
Deve-se desenvolver uma cultura institucional de desospitalização, definindo que a terapia nutricional é uma das atividades envolvidas no processo de cuidados com a alta hospitalar. Após a triagem nutricional, devem ser avaliados todos os pacientes que estão em risco e rapidamente implementar uma intervenção nutricional, que deve ser continuamente monitorada. O plano de cuidados nutricionais deve estar inserido no plano de alta, e as ações de educação devem envolver todas as etapas, como escolha da fórmula, uso de equipamento e demais cuidados envolvidos ${ }^{12,16,38,39}$. Os sobreviventes da internação em UTI, os pacientes submetidos a grandes cirurgias, os indivíduos mais idosos e aqueles com internações prolongadas devem ser considerados os candidatos mais importantes para estratégias de planejamento nutricional da alta. A depender da disponibilidade de recursos locais, tal planejamento pode ser estendido a pacientes de menor risco nutricional, especialmente quando se considera o impacto potencial da terapia nutricional em pacientes de enfermarias clínicas ${ }^{40}$.

Os pacientes hospitalizados são frequentemente despreparados para o autocuidado após a alta hospitalar, ocasionando, muitas vezes, a readmissão hospitalar não planejada. Cancino et al. ${ }^{13}$ elencaram as principais ações a serem realizadas para que o processo de alta hospitalar seja bem realizado. Os profissionais envolvidos no planejamento da alta devem procurar identificar barreiras de comunicação com a equipe de saúde, o paciente e seus cuidadores. Idealmente, deve-se determinar o melhor método de transmissão de informações conforme o nível de entendimento do paciente e de seus cuidadores, incluindo explicações verbais, demonstrações práticas e materiais audiovisuais, que devem sempre ser focados no paciente. Também é importante identificar a necessidade de reforço para melhor compreensão, sendo útil o emprego da técnica de teach back, segundo a qual o indivíduo sendo instruído explica o que the foi orientado. Por fim, a continuidade do cuidado e a gestão dos casos após a alta, por meio de apoio comunitário, visitas domiciliares, contato telefônico e acompanhamento ambulatorial, podem aumentar ainda mais as chances de sucesso terapêutico ${ }^{35}$. A elaboração de um relatório de transição e cuidado, a informação sobre sinais de alerta e o agendamento de visitas e exames de seguimento são pontos importantes para a continuidade do cuidado. Existem evidências de que o aconselhamento dietético individualizado por nutricionistas após a alta hospitalar melhora o peso corporal, bem como a ingestão energética e proteica em pacientes idosos com risco nutriciona ${ }^{41}$. Um estudo recente em pacientes que tiveram infarto agudo do miocárdio e apresentavam risco de desnutrição demonstrou que, quando comparados a pacientes que receberam cuidado padrão, pacientes randomizados para receber tratamento nutricional individualizado tiveram maior ingestão de energia e maior ganho funcional para realização das atividades diárias do que os randomizados para o grupo controle ${ }^{42}$.

A importância do atendimento pós-hospitalar é uma realidade. A European Society for Clinical Nutrition and 
Metabolism (ESPEN) acaba de publicar diretrizes para nutrição domiciliar; entre as várias recomendações, considera-se de extrema relevância a existência de uma equipe multiprofissional responsável por acompanhar, orientar e monitorizar os pacientes após a alta ${ }^{43}$. De maneira semeIhante, a Diretriz Brasileira de Terapia Nutricional Domiciliar também enfatiza a importância de se estabelecer uma transição segura do ambiente hospitalar para o domiciliar, o que inclui a recomendação do planejamento de alta e o estabelecimento de uma boa comunicação entre a equipe multiprofissional responsável pelo paciente no atendimento hospitalar e quem estará responsável pelo cuidado domici$\operatorname{liar}^{44}$. A elaboração e implementação de um plano de alta sistematizado é colocada como mandatória para pacientes com indicação de terapia nutricional domiciliar para que se assegure no ambiente domiciliar a continuidade da assistência do hospitalar. De acordo com as orientações, todas as possibilidades de terapia nutricional domiciliar - enteral, parenteral e suplemento oral - devem ser contempladas no plano de alta hospitalar. Para garantir a qualidade nutricional e microbiológica, a legislação brasileira em vigência (Resolução $N^{\circ}$ 63/2000) recomenda que os pacientes hospitalizados recebam apenas dietas enterais industrializadas ${ }^{45}$. Para atender a essa demanda, o mercado nacional conta com grande número de opções de fórmulas enterais, desenvolvidas para cumprir as exigências legais. Assim, equipara a terapia nutricional em nosso País com aquela feita em países desenvolvidos. Por outro lado, o parágrafo $3^{\circ}$ do artigo $8^{\circ}$ da Portaria № 120, de 2009, define que "as dietas artesanais e/ou semiartesanais deverão ser incentivadas naqueles pacientes sob cuidados e/ou internação domiciliar" ${ }^{\prime \prime 6}$.

Alguns estudos relatam que a dieta artesanal é mais natural, fisiológica, flexível e econômica, tendo também valor sentimental do prazer de se alimentar como o restante da família ${ }^{47-49}$. Entretanto, vários estudos demonstram que a dieta artesanal não atinge as necessidades calóricas reais, além de ser deficiente em nutrientes isolados ou agrupados, especialmente ferro ${ }^{50-52}$, cálcio ${ }^{50-53}$, magnésio e vitamina $C^{49,53}$.

Outro ponto negativo da dieta artesanal é a contaminação, cujo risco aumenta quanto maior a manipulação. Estudo de Medina et al..$^{54}$ demonstrou que houve contaminação em $68,7 \%$ das amostras de quatro tipos de dietas enterais artesanais feitas no domicílio, indicando que, do ponto de vista microbiológico, estas dietas não são tão seguras quanto as fórmulas industrializadas. Machado de Souza et al. ${ }^{55}$ analisaram a composição da dieta enteral artesanal, e a análise bromatológica isolada das fases demonstrou que a sopa teve baixo percentual de adequação para as proteínas ( $22 \%$ em relação ao calculado), o que refletiu negativamente na adequação proteica do plano dietético total ( $44 \%$ em relação ao calculado). Além disso, a sopa teve baixa concentração de carboidratos (53\% em relação ao calculado) e de lipídeos (50\% em relação ao calculado), o que resultou em inadequação do conteúdo energético total e desequilíbrio na distribuição de macronutrientes. Estes autores sugerem que as dietas enterais sejam fornecidas de forma mista, ou seja, elaboradas a partir da mistura de dietas comerciais somadas a alimentos in natura, já que há uma dificuldade em estabelecer a adequação nutricional de dietas unicamente artesanais ${ }^{55}$.

Os resultados de uma revisão sistemática sobre a frequência de uso e experiências de dietas caseiras em pacientes ou cuidadores e prestadores de cuidados de saúde demonstraram que as dietas caseiras devem ser indicadas para pacientes estáveis, com gastrostomia e com idade mínima de 6 meses, desde que não apresentem distúrbios metabólicos ou alergias alimentares múltiplas que proíbam alimentos integrais ${ }^{56}$. Os autores reforçam o treinamento e motivação do cuidador e envolvimento dos familiares, bem como as dificuldades para manipulação dos equipamentos e espaço para armazenamento quando se usam dietas artesanais, sendo fundamental o monitoramento e supervisão com nutricionista. Por outro lado, Johnson et al. ${ }^{57}$ comentam sobre a proibição do uso de dietas artesanais pelas empresas de home care americanas, pois as mesmas temem pela composição nutricional desconhecida da fórmula, oclusão da sonda nasoenteral, e aumento do risco de infecções. Por fim, as diretrizes da ESPEN para nutrição domiciliar consideram que as dietas artesanais são menos eficazes que as dietas industrializadas, não devendo ser utilizadas em pacientes em terapia nutricional enteral domiciliar, por serem menos seguras.

Não obstante à discussão acima, diversas barreiras podem ser identificadas na tentativa de coordenar esforços que possam garantir o adequado planejamento e a implementação da alta nutricional, especialmente em serviços com recursos ainda insuficientes. $\bigcirc$ Quadro 1 demonstra as principais barreiras identificadas pelo painel, juntamente com propostas para superá-las. As barreiras podem ter origem no paciente e na equipe de nutrição, mas a maior parte delas pode ser superada se forem reconhecidas e abordadas de maneira proativa e sistemática.

As estratégias para contornar tais barreiras envolvem a educação do paciente e cuidadores para aumentar a compreensão da importância de se alcançar as metas nutricionais e seguir as orientações para aumentar a adesão à terapia nutricional prescrita; educação continuada dos profissionais de saúde envolvidos no cuidado para que se estabeleçam todas etapas para a elaboração de um plano de alta efetivo; estratégias administrativas voltadas para identificar restrições e aprimorar o atendimento e garantir a organização de equipes multidisciplinares; e o acompanhamento após a alta. As barreiras identificadas na discussão do painel são consistentes com as levantadas por outros trabalhos da literatura $^{13,16,58}$. Um estudo conduzido na Dinamarca baseado em entrevistas com profissionais de saúde agrupou as barreiras para a terapia nutricional na transição hospital-casa em três esferas principais: educacionais (falta de conhecimento, treino e experiência), organizacionais (problemas na comunicação e falta de diretrizes e instruções locais) e econômicas (falta de tempo e recursos, tempo curto de internação) $)^{58}$. 
Quadro 1 - Barreiras frequentes identificadas pelos membros do painel e possiveis intervenções nos processos de alta hospitalar.

Barreiras Possíveis intervenções

Relacionadas ao paciente

Falta de entendimento da importância da terapia nutricional

Desenvolvimento de lâminas/vídeos/animações demonstrando a importância da alimentação para uso nos primeiros dias de internação

Adesivos para fixação em bandejas de alimentação oral e terapia nutricional oral com estímulo ao processo de nutrição

Dificuldades de comunicação

Indefinição do cuidador de referência

Problemas financeiros

Relacionadas à equipe de terapia nutricional

Ausência de Equipe Multidisciplinar de Terapia Nutricional (EMTN)

Ausência de triagem nutricional, diagnóstico nutricional e avaliação nutricional

Subindicação de terapia nutricional oral e da dieta enteral

Não entendimento do tempo necessário de uso de terapia nutricional oral

Terapia nutricional oral não custeada por convênios
Desenvolvimento de variadas linguagens de comunicação para alta hospitalar (vídeos, pdf para WhatsApp, quiz para tablets ou TVs com possibilidades de teach back)

Definição, nos primeiros dias de internação, do cuidador responsável de referência

Definição de rede de distribuição de suplementos na rede pública

Encaminhamentos para acompanhamento nutricional após alta hospitalar

Trabalho junto à administração hospitalar sobre os benefícios técnicos e financeiros da implementação de EMTN

Padronização entre nutricionistas e/ou enfermeiros de ferramentas de triagem a serem aplicadas

Realizar e registrar a avaliação e o diagnóstico nutricional, bem como o acompanhamento do estado nutricional

Checklist de critérios para indicação de terapia nutricional

Treinamento da equipe de terapia nutricional sobre necessidades nutricionais aumentadas mesmo após a alta hospitalar e a necessidade de suplementação a médio ou longo prazo em determinados pacientes

Comparativo de efetividade e preço entre terapia nutricional oral e alimentação padrão

Dieta Industrializada x Dieta Artesanal (caseira)

Negociação de terapia nutricional oral junto aos convênios

Baixo tempo disponível dos colaboradores

Padronização de rotinas

Desenvolvimento de ferramentas eficazes e rápidas para processos de alta hospitalar

Transição de terapia nutricional enteral para via oral sem avaliação da necessidade de terapia nutricional oral

Desenvolvimento de sugestão de fluxos de desmame de terapia nutricional enteral

Ausência de educação continuada de alta para familiares/pacientes

Realização de reconciliação medicamentosa

Definição de compatibilidades e uso de medicações via sonda

Treinamento sobre monitorização de fixação da sonda, cuidados para prevenção de obstrução

Ausência de plano multiprofissional de alta e início tardio da elaboração do plano

Altas compartilhadas

Estabelecer previsão da via de terapia nutricional para a alta

Início precoce do processo de alta hospitalar direcionado para a via prevista

Ausência de plano de alta para as altas realizadas em finais de semana e feriados

Garantir que o processo de planejamento efetivo de alta seja implementado mesmo em feriados e fins de semana

Não entendimento da importância da terapia nutricional a longo prazo após a alta hospitalar
Treinamentos focando na reabilitação muscular após a alta hospitalar

Melhor conhecimento da linha de produtos de terapia nutricional para uso ambulatorial

Importância do acompanhamento nutricional após a alta hospitalar (interromper o ciclo: "não atendo consultório, não encaminho")

Revendas acessíveis

Melhorar dificuldades em e-commerce 
Proposta de Instrumento para Alta Nutricional

\section{Planejada}

Com base na discussão precedente e na experiência dos autores, o instrumento apresentado na Figura 1 é proposto para auxiliar equipes de nutrição e outros profissionais de saúde no planejamento da alta nutricional. $O$ instrumento tem por objetivo sistematizar a alta nutricional por meio da coleta das informações mais relevantes ligadas ao risco nutricional e à abordagem terapêutica desse risco. Tais informações incluem aspectos relevantes da história médica recente durante a internação hospitalar, dados antropométricos, presença de critérios fenotípicos de desnutrição e características previstas no curto e médio prazos que podem influenciar a conduta, tais como sarcopenia e dependência funcional, e as modalidades de atendimento do paciente em seu domicílio ou serviços de saúde. Por fim, o instrumento apresenta checklists que têm por objetivo organizar as atividades a serem realizadas e normatizar a comunicação com o paciente, seus cuidadores e a equipe de saúde.

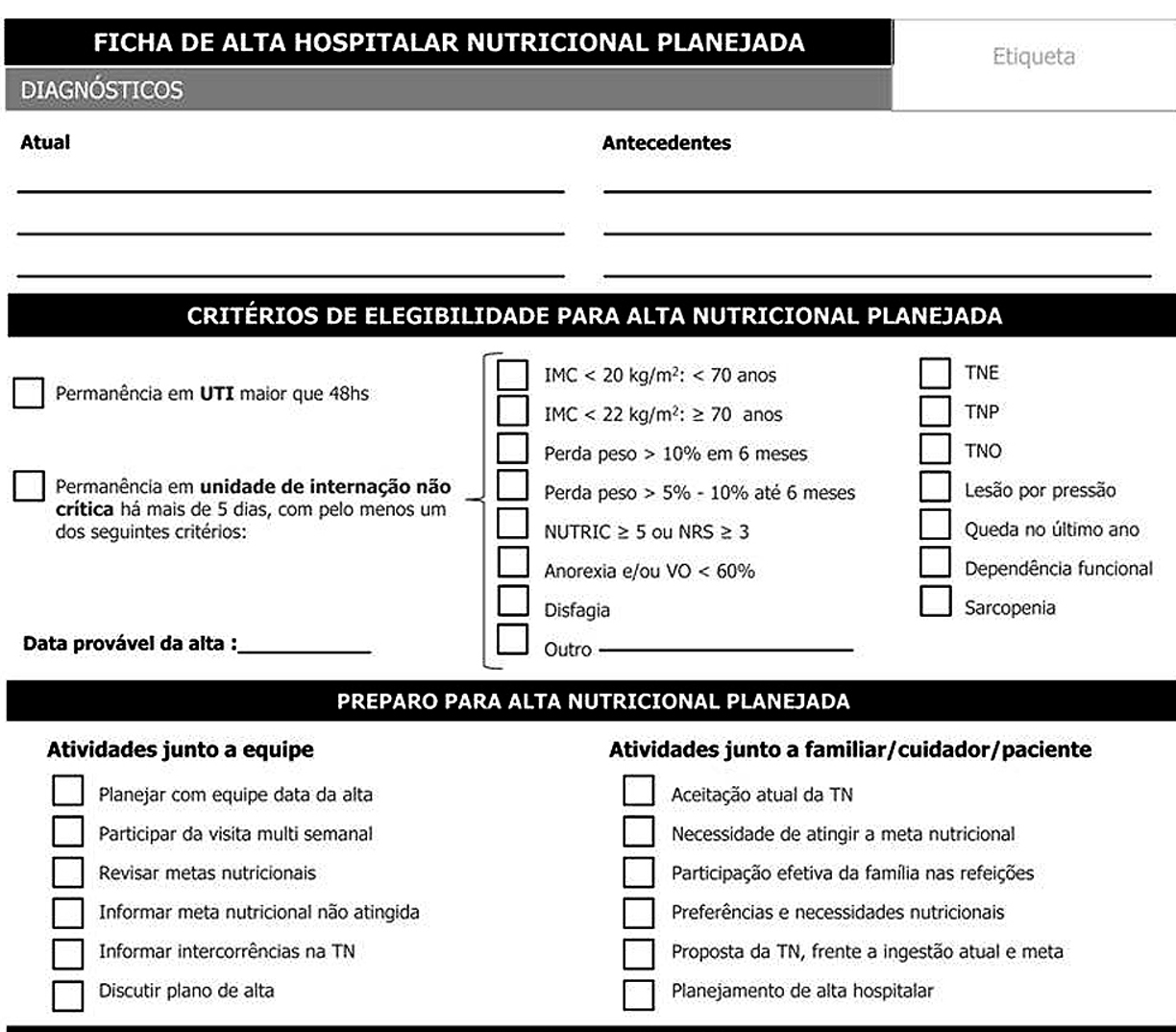

TN NA ALTA HOSPITALAR

vo exclusiva
Com adequação de
consistência
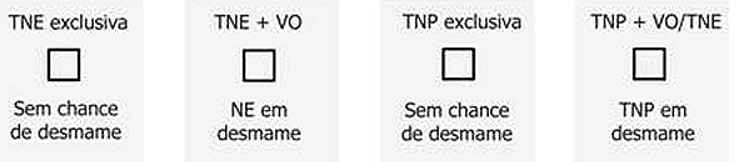

OBSERVAÇÕES:

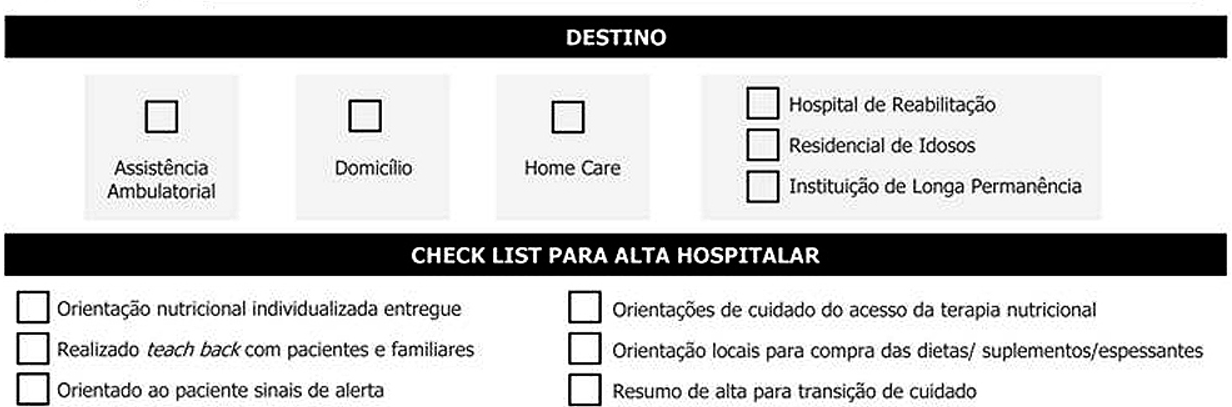

DATA DA ALTA :

CONTATO TELEFônICO PÓS ALTA:

Figura 1 - Proposta de instrumento para planejamento nutricional na alta hospitalar. 
Com relação aos critérios de elegibilidade para utilização do plano, a proposta é que o instrumento seja utilizado para auxiliar o planejamento da transição da UTI - Unidade de Internação Casa para pacientes em risco nutricional por: 1) permanência em UTI por período maior do que 48 horas ou 2) permanência em unidades de internação não críticas há mais de 5 dias com presença de ao menos um dos critérios detalhados na Figura 1. Tais critérios levam em conta o índice de massa corporal (IMC) e a categoria de idade, a faixa de perda de peso, escore NUTRIC $\geq 5$ ou NRS $\geq 3$, anorexia e/ou $\mathrm{VO}<60 \%$, disfagia, uso de terapia nutricional enteral, parenteral, ou oral, lesão por pressão, queda no ano anterior, dependência funcional, sarcopenia, ou alguma outra condição que na avaliação da equipe possa oferecer risco nutricional ${ }^{35,44,59,60}$.

Em termos práticos, a abertura do instrumento será realizada pelo nutricionista que recebe o paciente proveniente da UTI ou que acompanha o paciente que está mais de 5 dias na unidade de internação não crítica e que apresenta ao menos um dos critérios listados. A abertura deve ser feita já no momento da admissão ou o mais precoce possível, para que os pacientes em risco sejam colocados em alta nutricional planejada. Uma data provável para a alta deve ser estabelecida logo no início do plano. A partir de sua abertura, o instrumento traz uma série de atividades de preparo para a alta que devem ser realizadas junto à equipe que assiste o paciente, bem como atividades junto ao próprio paciente e seus familiares/cuidadores, para garantir uma alta nutricional segura. As estratégias de comunicação devem ser adequadas às necessidades dos pacientes, por exemplo, no caso de pacientes com necessidade especial (auditiva/visual) que preencham os critérios de elegibilidade para a alta nutricional planejada.A seção "TN na alta hospitalar" é destinada à coletada de informações sobre a terapia nutricional orientada na alta. A terapia nutricional será definida de acordo com as necessidades e condições do paciente, seguindo as recomendações ${ }^{44}$. No caso de terapia nutricional oral, a consistência dos alimentos deverá ser adequada conforme a condição do paciente e grau disfagia, quando presente, seguindo a orientação do nutricionista e/ou fonoaudiólogo, em conformidade com as diretrizes ${ }^{61}$. Pacientes com disfagia serão orientados, também, sobre a necessidade ou não do uso de espessantes.

O instrumento coletará também informações sobre o destino/local de cuidado pós-alta (casa ou outra instituição) e a necessidade de assistência ambulatorial ou home care. Por fim, na última seção, há um check list das atividades finais a serem realizadas junto ao paciente para a alta hospitalar, que incluem a entrega de orientação nutricional individualizada, a confirmação do entendimento do paciente com relação à sua condição, às orientações e cuidados necessários no ambiente domiciliar, incluindo o teach back, orientações sobre sinais de alerta, cuidados com o acesso e locais para compra de dietas, suplementos e espessantes, e entrega do resumo de alta, bem como campos para registro da data efetiva da alta e da data prevista para realização do primeiro contato telefônico de acompanhamento após a alta, para que sirvam como base para contatos telefônicos ou atendimento ambulatorial a posteriori e verificação se no período houve interrupção da terapia nutricional e/ou reinternação.

Para cada paciente, apenas uma única ficha será aberta, devendo ser preenchida/atualizada durante todo o período de acompanhamento. Dessa forma, o instrumento permitirá que o serviço colete de modo sistematizado informações sobre o diagnóstico e antecedentes, os critérios que o levaram a ser elegível para a alta nutricional planejada, modalidade de terapia nutricional indicada na alta, e também funcionará como check-list para que a equipe acompanhe e se certifique de que todas as etapas listadas no plano foram executadas para uma alta segura. É fundamental que a segurança da alta seja garantida também nos casos de alta durante finais de semana ou feriados. No momento da alta, é importante que o paciente esteja acompanhado de um familiar ou cuidador, especialmente, nos casos de pacientes idosos e neuropatas.

\section{DISCUSSÃO}

Diversos fatores institucionais e pessoais podem interferir e dificultar a tentativa de oferecer reabilitação nutricional e uma transição segura na trajetória que frequentemente envolve o ambiente de UTI, a enfermaria e o domicílio do paciente. A atenção a esses fatores é uma medida essencial na tentativa de prover uma alta hospitalar segura, que pode aumentar as chances de sucesso terapêutico e reduzir o risco de reinternação. Certamente existem dificuldades em todas as etapas, incluindo na transição da UTI para a enfermaria; neste caso, a falta de continuidade entre diferentes serviços e a troca da equipe profissional podem representar barreiras de difícil transposição conforme a natureza do serviço. Esforços devem ser empreendidos para que essas barreiras sejam contornadas, na tentativa de propor reabilitação nutricional efetiva a médio e longo prazos. Além disso, é importante considerar que a efetividade dessa estratégia também depende da capacidade de acompanhar os pacientes após a alta e monitorar eventuais reinternações. Em estudo realizado com idosos desnutridos, verificou-se que o planejamento da alta hospitalar com acompanhamento nutricional antes e após a alta teve um pequeno impacto nos parâmetros nutricionais e funcionais, mas não nos desfechos clínicos ${ }^{14}$.

Uma das medidas que podem representar um fio condutor na tentativa de superar esses desafios é a criação de mecanismos institucionais para planejamento efetivo da 
alta nutricional. Para tanto, pode ser útil a adoção de instrumentos como aquele aqui proposto que pretende facilitar as condutas por parte da equipe de nutrição e de todos os profissionais de saúde preocupados em garantir os melhores resultados possíveis para pacientes com risco nutricional. $\bigcirc$ instrumento aqui apresentado deverá ser testado na prática clínica, com intuito de verificar sua eficácia como ferramenta auxiliar no processo de alta e acompanhamento nutricional do paciente. Destaque especial deve ser dado aos itens do bloco "preparo para alta nutricional planejada", no qual a comunicação entre os diferentes profissionais envolvidos no processo da alta hospitalar é fundamental para o sucesso do evento. Outro ponto importante é o acompanhamento após a alta, aqui sugerido como "contatos telefônicos pósalta". A partir desses contatos é possível verificar se as metas estabelecidas estão sendo cumpridas e, até mesmo, se houve reinternação ou qualquer outra intercorrência. Desse modo, espera-se que os profissionais tenham um instrumento para melhor acompanhamento da jornada do paciente, conduzindo para a alta hospitalar bem-sucedida.

\section{REFERÊNCIAS}

1. McWhirter JP, Pennington CR. Incidence and recognition of malnutrition in hospital. BMJ. 1994;308(6934):945-8.

2. Norman K, Pichard C, Lochs H, Pirlich M. Prognostic impact of disease-related malnutrition. Clin Nutr. 2008;27(1):5-15.

3. Correia MITD, Perman MI, Waitzberg DL. Hospital malnutrition in Latin America: a systematic review. Clin Nutr. 2017;36(4):958-67.

4. Lim SL, Ong KC, Chan YH, Loke WC, Ferguson M, Daniels L. Malnutrition and its impact on cost of hospitalization, length of stay, readmission and 3-year mortality. Clin Nutr. 2012;31(3):345-50.

5. Hudson L, Chittams J, Griffith C, Compher C. Malnutrition identified by Academy of Nutrition and Dietetics/American Society for Parenteral and Enteral Nutrition is associated with more 30-day readmissions, greater hospital mortality, and longer hospital stays: a retrospective analysis of nutrition assessment data in a major medical center. JPEN J Parenter Enteral Nutr. 2018;42(5):892-7.

6. Correia MI, Waitzberg DL. The impact of malnutrition on morbidity, mortality, length of hospital stay and costs evaluated through a multivariate model analysis. Clin Nutr. 2003;22(3):235-9.

7. Lew CCH, Yandell R, Fraser RJL, Chua AP, Chong MFF, Miller $\mathrm{M}$. Association between malnutrition and clinical outcomes in the intensive care unit: a systematic review. JPEN J Parenter Enteral Nutr. 2017;41(5):744-58.

8. Fontes D, Generoso SV, Correia MITD. Subjective global assessment: a reliable nutritional assessment tool to predict outcomes in critically ill patients. Clin Nutr. 2014;33(2):291-5.

9. Vallejo KP, Martinez CM, Adames AAM, Fuchs-Tarlovsky V, Nogales GCC, Paz RER, et al. Current clinical nutrition practices in critically ill patients in Latin America: a multinational observational study. Crit Care. 2017;21(1):227.
10. Lima J, Teixeira PP, Eckert IDC, Burgel CF, Silva FM. Decline of nutritional status in the first week of hospitalisation predicts longer length of stay and hospital readmission during 6-month follow-up. Br J Nutr. 2020:1-8.

11. Baker EB, Wellman NS. Nutrition concerns in discharge planning for older adults: a need for multidisciplinary collaboration. J Am Diet Assoc. 2005;105(4):603-7.

12. Tappenden KA, Quatrara B, Parkhurst ML, Malone AM, Fanjiang G, Ziegler TR. Critical role of nutrition in improving quality of care: an interdisciplinary call to action to address adult hospital malnutrition. Medsurg Nurs. 2013;22(3):147-65.

13. Cancino RS, Manasseh C, Kwong L, Mitchell SE, Martin J, Jack BW. Project RED impacts patient experience. J Patient Exp. 2017;4(4):185-90.

14. Young AM, Mudge AM, Banks MD, Rogers L, Demedio K, Isenring E. Improving nutritional discharge planning and follow up in oldermedical inpatients: hospital to home outreach for malnourished elders. Nutr Diet. 2018;75(3):283-90.

15. Ingstad K, Uhrenfeldt L, Kymre IG, Skrubbeltrang C, Pedersen P. Effectiveness of individualised nutritional care plans to reduce malnutrition during hospitalisation and up to 3 months post-discharge: a systematic scoping review. BMJ Open. 2020;10(11):e040439.

16. Mennuni M, Gulizia MM, Alunni G, Amico AF, Bovenzi FM, Caporale R, et al. ANMCO position paper: hospital discharge planning: recommendations and standards. Eur Heart J Suppl. 2017;19(Suppl D):D244-55.

17. Gonçalves-Bradley DC, Lannin NA, Clemson LM, Cameron ID, Shepperd S. Discharge planning from hospital. Cochrane Database Syst Rev. 2016(1):CD000313.

18. Naylor MD, Brooten D, Campbell R, Jacobsen BS, Mezey $\mathrm{MD}$, Pauly MV, et al. Comprehensive discharge planning and home follow-up of hospitalized elders: a randomized clinical trial. JAMA. 1999;281(7):613-20.

19. Lainscak M, Kadivec S, Kosnik M, Benedik B, Bratkovic M, Jakhel T, et al. Discharge coordinator intervention prevents hospitalizations in patients with COPD: a randomized controlled trial. J Am Med Dir Assoc. 2013;14(6):450. e1-6.

20. van Mol M, Nijkamp M, Markham C, Ista E. Using an intervention mapping approach to develop a discharge protocol for intensive care patients. BMC Health Serv Res. 2017; 17(1):837.

21. Waitzberg DL, Caiaffa WT, Correia MI. Hospital malnutrition: the Brazilian national survey (IBRANUTRI): a study of 4000 patients. Nutrition. 2001;17(7-8):573-80.

22. Pinho NB, Martucci RB, Rodrigues VD, D'Almeida CA, Thuler LCS, Saunders C, et al. High prevalence of malnutrition and nutrition impact symptoms in older patients with cancer: results of a Brazilian multicenter study. Cancer. 2020;126(1):156-64.

23. Mullin GE, Fan L, Sulo S, Partridge J. The association between oral nutritional supplements and 30-day hospital readmissions of malnourished patients at a US Academic Medical Center. J Acad Nutr Diet. 2019;119(7):1168-75.

24. Kirkland LL, Shaughnessy E. Recognition and prevention of nosocomial malnutrition: a review and a call to action! Am J Med. 2017;130(12):1345-50.

25. Mowe M, Bosaeus I, Rasmussen HH, Kondrup J, Unosson M, Rothenberg E, et al; Scandinavian Nutrition Group. Insufficient nutritional knowledge among health care workers? Clin Nutr. 2008;27(2):196-202. 
26. Bavelaar JW, Otter CD, van Bodegraven AA, Thijs A, van Bokhorst-de van der Schueren MA. Diagnosis and treatment of (disease-related) in-hospital malnutrition: the performance of medical and nursing staff. Clin Nutr. 2008;27(3):431-8.

27. Volkert D, Saeglitz C, Gueldenzoph H, Sieber CC, Stehle P. Undiagnosed malnutrition and nutrition-related problems in geriatric patients. J Nutr Health Aging. 2010;14(5):387-92.

28. Ter Beek L, Vanhauwaert E, Slinde F, Orrevall Y, Henriksen C, Johansson M, et al. Unsatisfactory knowledge and use of terminology regarding malnutrition, starvation, cachexia and sarcopenia among dietitians. Clin Nutr. 2016;35(6):1450-6.

29. Fjeldstad SH, Thoresen L, Mowé M, Irtun O. Changes in nutritional care after implementing national guidelines-a 10-year follow-up study. Eur J Clin Nutr. 2018;72(7):1000-6.

30. Merriweather J, Smith P, Walsh T. Nutritional rehabilitation after ICU - does it happen: a qualitative interview and observational study. J Clin Nurs. 2014;23(5-6):654-62.

31. Volkert D, Beck AM, Cederholm T, Cruz-Jentoft A, Goisser $\mathrm{S}$, Hooper L, et al. ESPEN guideline on clinical nutrition and hydration in geriatrics. Clin Nutr. 2019;38(1):10-47.

32. Leij-Halfwerk S, Verwijs MH, van Houdt S, Borkent JW, Guaitoli PR, Pelgrim T, et al; MaNuEL Consortium. Prevalence of protein-energy malnutrition risk in European older adults in community, residential and hospital settings, according to 22 malnutrition screening tools validated for use in adults $\geq 65$ years: a systematic review and meta-analysis. Maturitas. 2019;126:80-9.

33. Singer P, Blaser AR, Berger MM, Alhazzani W, Calder PC, Casaer MP, et al. ESPEN guideline on clinical nutrition in the intensive care unit. Clin Nutr. 2019;38(1):48-79.

34. Correia MI, Hegazi RA, Higashiguchi T, Michel JP, Reddy BR, Tappenden KA, et al. Evidence-based recommendations for addressing malnutrition in health care: an updated strategy from the feedM.E. Global Study Group. J Am Med Dir Assoc. 2014;15(8):544-50.

35. Toledo DO, Piovacari SMF, Horie LM, Matos LBN, Castro MG, Ceniccola GD, et al. Campanha "Diga não à desnutrição": 11 passos importantes para combater a desnutrição hospitalar. BRASPEN J. 2018;33(1):86-100.

36. Ramsay P, Huby G, Merriweather J, Salisbury L, Rattray J, Griffith D, et al. Patient and carer experience of hospitalbased rehabilitation from intensive care to hospital discharge: mixed methods process evaluation of the RECOVER randomised clinical trial. BMJ Open. 2016;6(8):e012041.

37. Usher MG, Fanning C, Wu D, Muglia C, Balonze K, Kim D, et al. Information handoff and outcomes of critically ill patients transferred between hospitals. J Crit Care. 2016;36:240-5.

38. Shepperd S, McClaran J, Phillips CO, Lannin NA, Clemson LM, McCluskey A, et al. Discharge planning from hospital to home. Cochrane Database Syst Rev. 2010;(1):CD000313.

39. Associação Nacional de Hospitais Privados. Núcleo Nacional das Empresas de Serviços de Atenção Domiciliar. Manual de Atenção Domiciliar. [Acesso em 05/09/2019]. Disponível em: http://www.neadsaude.org.br

40. Schuetz P, Fehr R, Baechli V, Geiser M, Deiss M, Gomes $\mathrm{F}$, et al. Individualised nutritional support in medical inpatients at nutritional risk: a randomised clinical trial. Lancet. 2019;393(10188):2312-21.

41. Munk T, Tolstrup U, Beck AM, Holst M, Rasmussen HH, Hovhannisyan K, et al. Individualised dietary counselling for nutritionally at-risk older patients following discharge from acute hospital to home: a systematic review and metaanalysis. J Hum Nutr Diet. 2016;29(2):196-208.

42. Otsuki I, Himuro N, Tatsumi H, Mori M, Niiya Y, Kumeta $\mathrm{Y}$, et al. Individualized nutritional treatment for acute stroke patients with malnutrition risk improves functional independence measurement: a randomized controlled trial. Geriatr Gerontol Int. 2020;20(3):176-82.

43. Bischoff SC, Austin P, Boeykens K, Chourdakis M, Cuerda C, Jonkers-Schuitema C, et al. ESPEN guideline on home enteral nutrition. Clin Nutr. 2020;39(1):5-22.

44. Van Aanholt DPJ, Matsuba CST, Dias MCG, Silva MLT, Campos ACL, Aguilar-Nascimento JE. Diretriz Brasileira de Terapia Nutricional Domiciliar. BRASPEN J. 2018;33(Supl 1):37-46

45. BRASIL. Agência Nacional de Vigilância Sanitária (Anvisa). Regulamento No 63 de 6 de julho de 2000. Aprova o Regulamento técnico para fixar os requisites mínimos exigidos para a Terapia Nutricional Enteral. Diário Oficial da União. Brasília: Ministério da Saúde. [Acesso em 13/04/2019]. Disponível em: http://www.sbnpe.com.br/resolucao-da-diretoria-colegiada-rdc-no-63-de-06-de-julho-de-2000

46. BRASIL. Ministério da Saúde, Secretaria de Atenção à Saúde. Portaria $\mathrm{N}^{\mathrm{o}} 120$, de 14 de abril de 2009. Assistência de alta complexidade de terapia nutricional. Diário Oficial da União. Brasília: Ministério da Saúde; 2009. [Acesso em 05/10/2019]. Disponível em: http://bvsms.saude.gov.br/bvs/saudelegis/ sas/2009/prt0120_14_04_2009.html

47. Mokhalalati JK, Druyan ME, Shott SB, Comer GM. Microbial, nutritional and physical quality of commercial and hospital prepared tube feedings in Saudi Arabia. Saudi Med J. 2004;25(3):331-41.

48. Sullivan MM, Sorreda-Esguerra P, Platon MB, Castro CG, Chou NR, Shott S, et al. Nutritional analysis of blenderized enteral diets in the Philippines. Asia Pac J Clin Nutr. 2004;13(4):385-91.

49. Cunha SFC, Borghi R, Dias MCG. Dietas enterais artesanais e semiartesanais: vantagens e desvantagens. In: Waitzberg DL, ed. Nutrição oral, enteral e parenteral na prática clínica. $5^{\mathrm{a}}$ ed. São Paulo: Atheneu; 2017.

50. Menegassi B, Sant'ana LS, Coelho JC, Martins AO, Pinto JPAN, Braga-Costa TM, et al. Características físico-químicas e qualidade nutricional de dietas enterais não-industrializadas. Rev Alimentos e Nutrição. 2007;18:127-32.

51. Borghi R, Araujo TD, Vieira RI, Theodoro de Souza T, Waitzberg DL. ILSI Task Force on enteral nutrition; estimated composition and costs of blenderized diets. Nutr Hosp. 2013;28(6):2033-8.

52. Lima VS, Souza FCA, Aguiar JPL, Yuyama LKO. Composição nutricional de dieta enteral artesanal a partir de alimentos convencionais do Município de Coari, Estado do Amazonas, Brasil. Rev Pan-Amaz Saude. 2015;6(2):29-36.

53. Bento APL. Elaboração de dietas enterais manipuladas, análise de sua composição nutricional e qualidade microbiológica [Dissertação de Mestrado]. Ribeirão Preto: Universidade de São Paulo, Faculdade de Medicina de Ribeirão Preto; 2010.

54. Medina JM, Nascimento GGF, Oliveira MRM. Contaminação microbiológica de dietas enterais. Rev Bras Nutr Clin. 2008;23:262-9.

55. Machado de Sousa LR, Rodrigues Ferreira SM, Madalozzo Schieferdecker ME. Physicochemical and nutritional 
characteristics of handmade enteral diets. Nutr Hosp. 2014;29(3):568-74.

56. Carter H, Johnson K, Johnson TW, Spurlock A. Blended tube feeding prevalence, efficacy, and safety: what does the literature say? J Am Assoc Nurse Pract. 2018;30(3):150-7.

57. Johnson TW, Milton DL, Johnson K, Carter H, Hurt RT, Mundi MS, et al. Comparison of microbial growth between commercial formula and blenderized food for tube feeding. Nutr Clin Pract. 2019;34(2):257-63.

58. Holst M, Rasmussen HH. Nutrition therapy in the transition between hospital and home: an investigation of barriers. J Nutr Metab. 2013;2013:463751.
59. Kaiser MJ, Bauer JM, Ramsch C, Uter W, Guigoz Y, Cederholm T, et al; MNA-International Group. Validation of the Mini Nutritional Assessment short-form (MNA-SF): a practical tool for identification of nutritional status. J Nutr Health Aging. 2009;13(9):782-8.

60. Hanna JS. Sarcopenia and critical illness: a deadly combination in the elderly. JPEN J Parenter Enteral Nutr. 2015;39(3):273-81.

61. Sociedade Brasileira de Geriatria e Gerontologia (SBGG). Diretoria SBGG Nacional. I consenso brasileiro de nutrição e disfagia em idosos hospitalizados. Barueri: Minha-Editora; 2011.

Local de realização do estudo: Danone Nutricia, São Paulo, SP, Brasil.

Conflito de interesse: RC declarou ter recebido honorários de consultoria científica da empresa Danone Nutricia na produção do presente artigo. NLAL declarou ter recebido honorários de consultoria da empresa Danone Nutricia como verba de pesquisa para realização de discussões e estudos relacionados ao presente artigo. ACSON declarou ter recebido honorários de consultoria da empresa Danone Nutricia. Os demais autores declararam não haver conflitos de interesse. 